\begin{tabular}{ccc}
\hline & International Journal of Engineering \& Technology, $7(2.12)(2018) 128-131$ \\
SPC & International Journal of Engineering \& Technology \\
Website: $w w w . s c i e n c e p u b c o . c o m / i n d e x . p h p / I J E T$ & Research Paper \\
\hline
\end{tabular}

\title{
Construction of remote control for web based street light pole
}

\author{
Yong Mo Kim * \\ Dept. of Visual Image Design, Gwangju University,277Hyodeok-ro, Nam-guGwangju city, 61743 , KOREA \\ *Corresponding author E-mail: Mdc2000@gwangju.ac.kr
}

\begin{abstract}
Background/Objectives: It is not easy to ascertain a maximum electricity production in case of solar energy generation and there is problem not to recognize failures for a long time even iffailures occurred in facility. Measures need to be taken not only to check irregularities and replacement time but to maximize overall efficiency of solar energy generation system.

Methods/Statistical analysis: It is necessary to build a control system based on web to analysis of various statistics information and real time index visibility. It is also necessary to verify SMS notification in the number of lightning in case of the falling of a thunderbolt, date and time of the occurrence, and correct operation of circuit breaker in the connection board.

Findings: It is needed to be introduced the system which not only considers excessive communication cost as well as failure treatment of communication device and replacement cost but also makes full use of a dispersed generating system. Control system which is able to do scientific action of making up for fault and proper monitoring as well as maintenance in a solar power plant is constructed in this study. Improvements/Applications: Smart control system that electricity generation is stopped or reactivated in case of emergency through making an autonomous judgment of difficulties or faulted conditions will be extended. Technology of new renewable energy such as wind power and geothermal power will be applied through enhanced design technology of communication interface.
\end{abstract}

Keywords: Monitoring; Streetlight; Solar Power Plant; Lightning; RTU

\section{Introduction}

There will be usually a cloudy weather, a typhoon, and an earthquake which makes generating efficiency less effective in operating a solar power plant. In consideration of foreign cases, a damage caused by a thunderbolt breaks out frequently [1], [2]. A solar power plant is a prime target for thunderbolt because high-standing structures which is able to block out sunlight is intentionally avoided or is removed in case of a flat installation of a solar power plant on a large scale in a vast extent of land. Monitoring system to simply not only accumulate amount of power but also identify an inverter disorder rather than a scientific monitoring and maintenance can be supplied by an inverter manufactures [3], [4]. Installation of communication devices or connection board in the unit structure makes generally invest in a lot of time to solve communication disorder rather than maintenance in a power plant. In order to be properly operate, it is needed to be introduced the system which not only considers excessive communication cost as well as failure treatment of communication device and replacement cost but also makes full use of a dispersed generating system. Control system which is able to do scientific action of making up for fault and proper monitoring as well as maintenance in a solar power plant is constructed in this study [5], [6].

\section{Related research}

Monitoring of solar energy generation system provides information which is able to ascertain conditions of solar energy generation in real time and performance evaluation through monitoring has been studied continuously. In the study on fault detection in solar energy generation system based on Wavelet conversion, the method of fault detection has problem to require redesign in case of changing specification with rising in unit cost. In order to solve the problem, the way to recognize type of failures and parts is adopted as Wavelet coefficient change earned by mapping of state variableslike invertor current or voltage is detected. Fault diagnosis in Wavelet coefficient change using normalized standard deviation is evaluated [seven, 8]. Domestic some enterprises are expanding markets overseas through developing own technology and achieving foreign certification more than introduction of advanced foreign technology. Study on reliability improvementthrough data analysis, capture technology data required not only to build lighting protection system but to need research and developmentis implemented. These enterprises will take new opportunities in market according to global market growth of lightning prevention $[9,10]$.

\section{Construction of remote control for web based streetlight pole}

\subsection{Development of SPD counter}

Collected data through Zigbee communications can be sent to the server and provides the following functions; circuit protection from lightning, checking conducting state of switchboard, and collecting lightning data such as the number of lightning, date and time of the occurrence.System configuration of SPD counter is shown in Figure 1. 


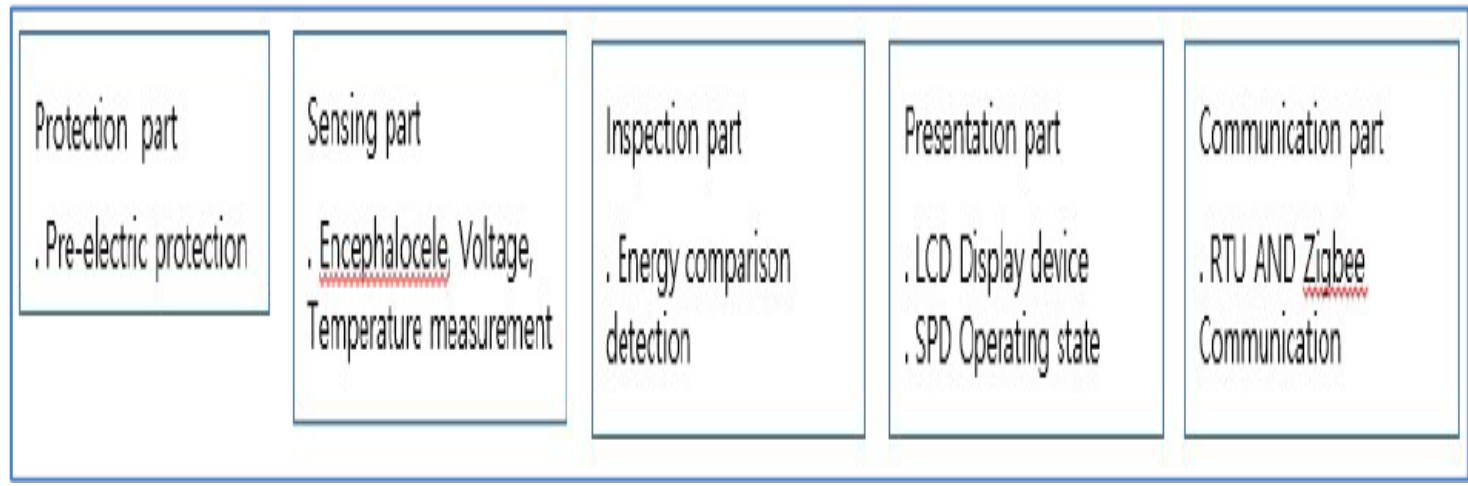

Fig. 1: System Configuration of SPD Counter.

Output of circuit drawing is shown in Figure 2 and results is shown in figure 3.

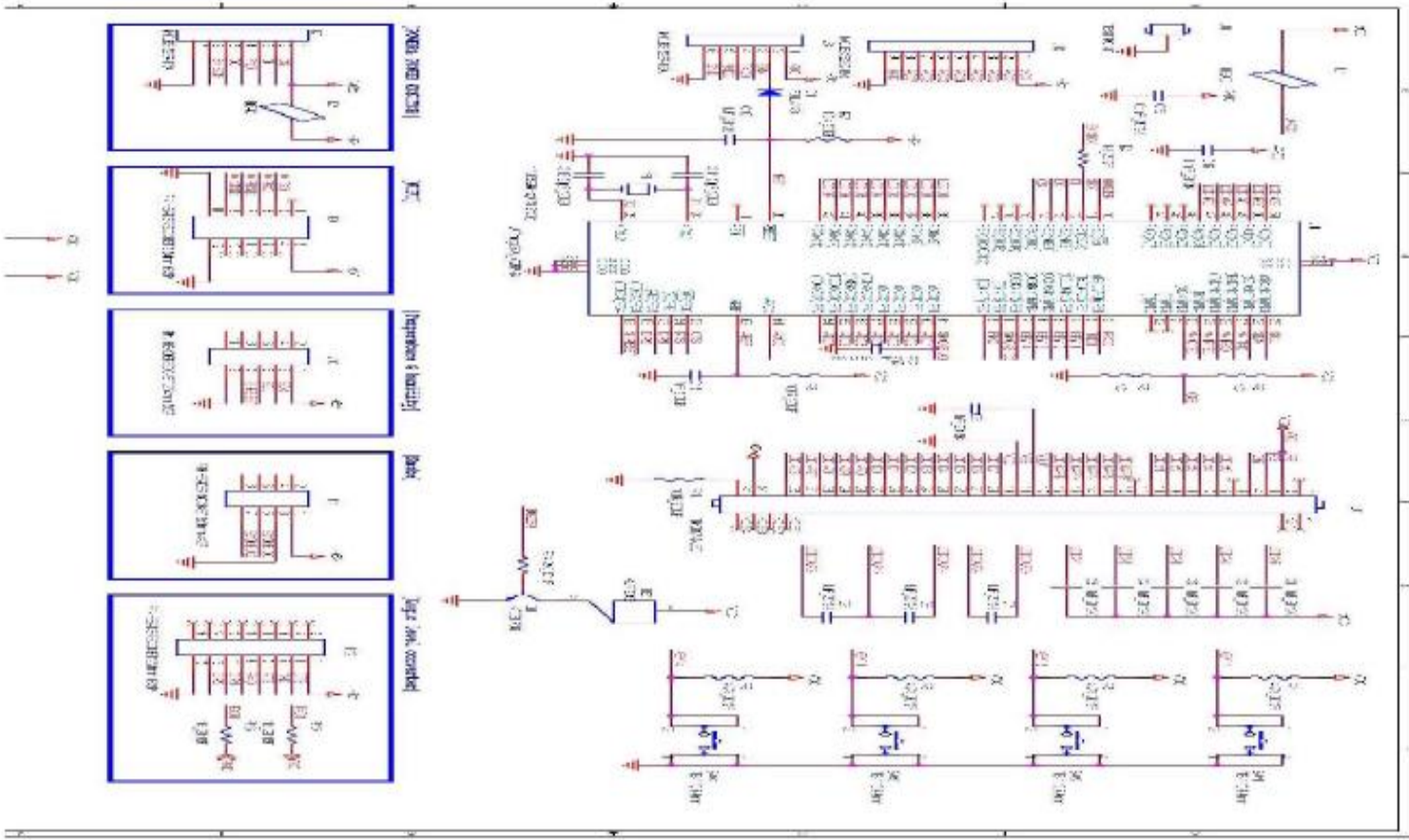

Fig. 2:.Circuit Design of SPD Counter.

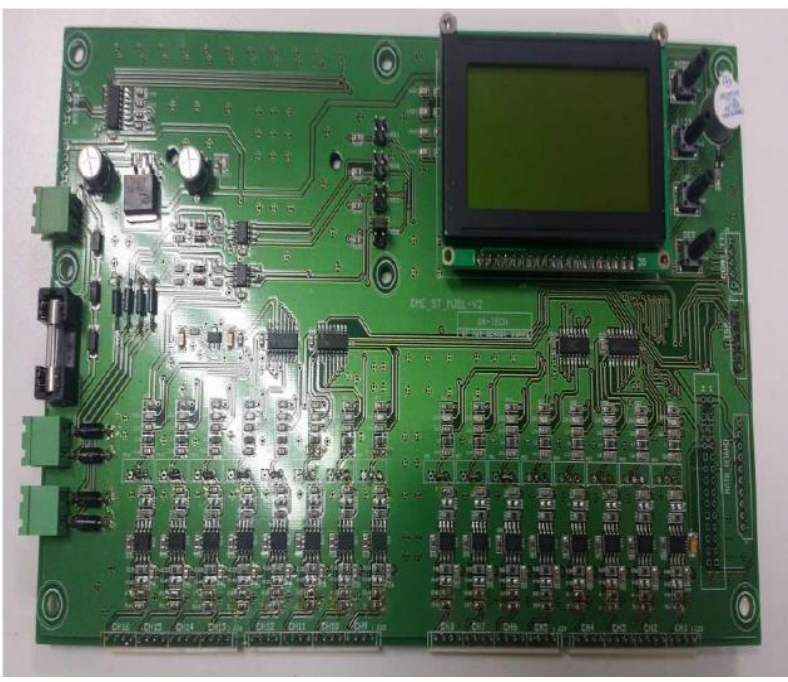

Fig. 3:.Output of SPD Counter Circuit.

\subsection{Manufacture of communication module zigbee}

It is conducted that circuit design of wireless communication module Zigbee to communicate between RTU and SPD and design of communication protocol composed of Mesh network topology. Major specification is as in the following; TCP communication is above $10 \mathrm{Mbps}$, Client Mode Operation, Zigbee communication is above $99 \%$ of receiving rate in broadcast. Communication protocol is STX + receiving device $(2)+$ transmitting device $(1)+$ connection boardID + Command + STRING1 STRING(n) + ETX + CRC + EOT, and error detecting code is 16bit CRC.

Circuit drawing and its product are shown in Figure 4 and 5, respectively. 


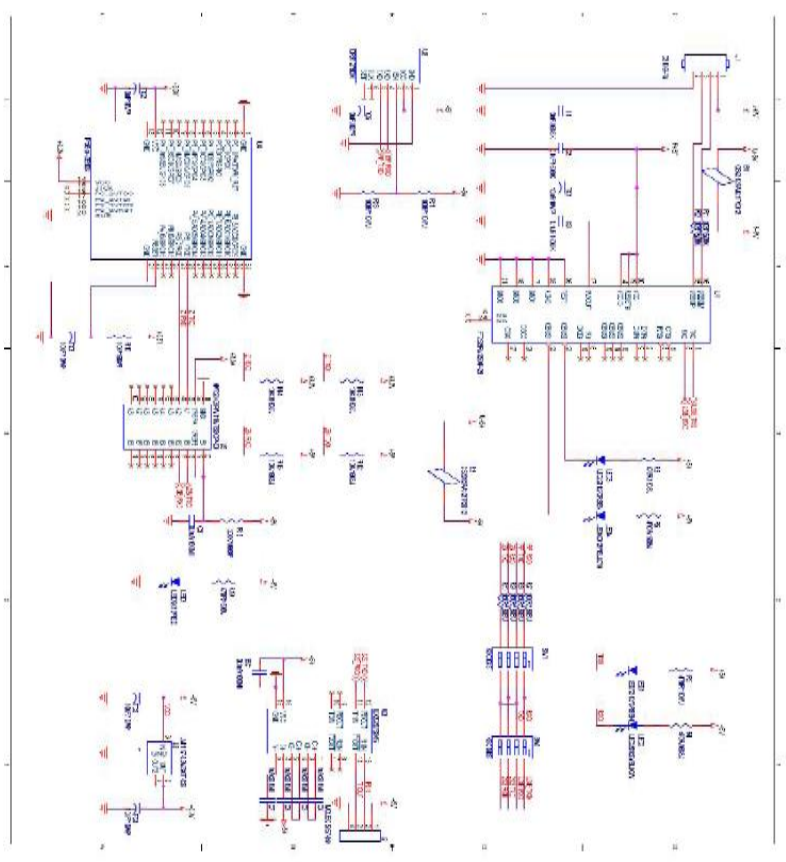

Fig. 4: Circuit Diagram of Zigbee Communication Module.

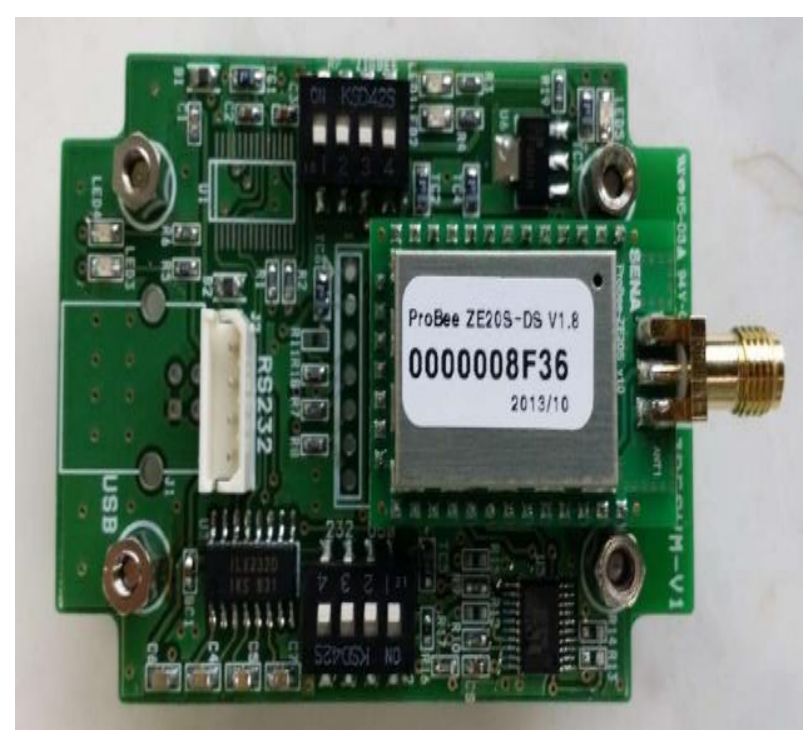

Fig. 5: Output of Zigbee Communication Module.

\subsection{Manufacture of data RTU}

Collected data from device and sensor is transmitted to remote supervisory and control system located in central monitor room using wire and radio communication equipment and its communication lines and received commend from remote supervisory and control system performs control online in real time. It is considered compatibility and expandability not only to know state of VCB/ACB relay but to be able to remote control of it in case of necessity. Major functions are state receiving which is connected by protective device of system connection as well as Modbus communication and its control, checking the shut off status of VCB/ACB and implementation of making and breaking, SMS notification to registered manager through embedded module in case of failure, and integration of monitoring data that is connected to DB server and TCP/IP. System diagram of RTU is shown in Figure 6, a circuit diagram and product of test bed is shown in Figure 7 and 8 respectively.

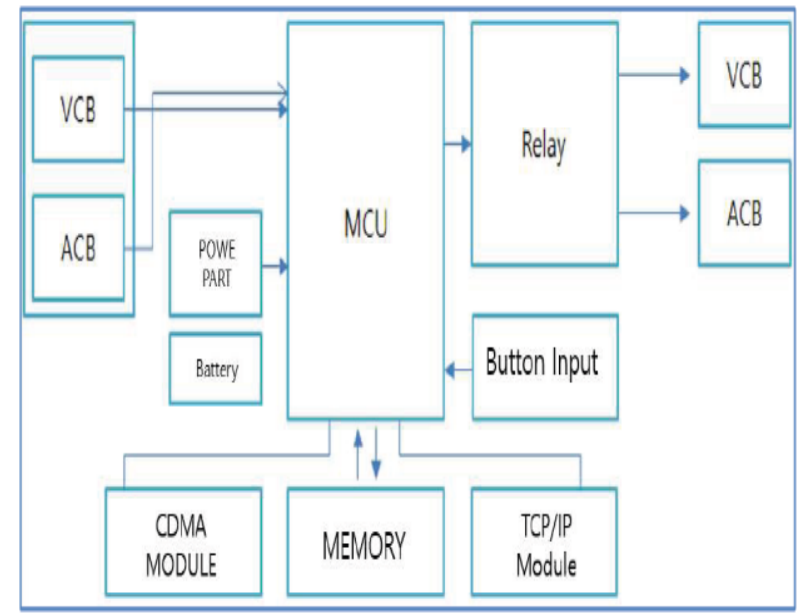

Fig. 6: Rtu System Diagram.

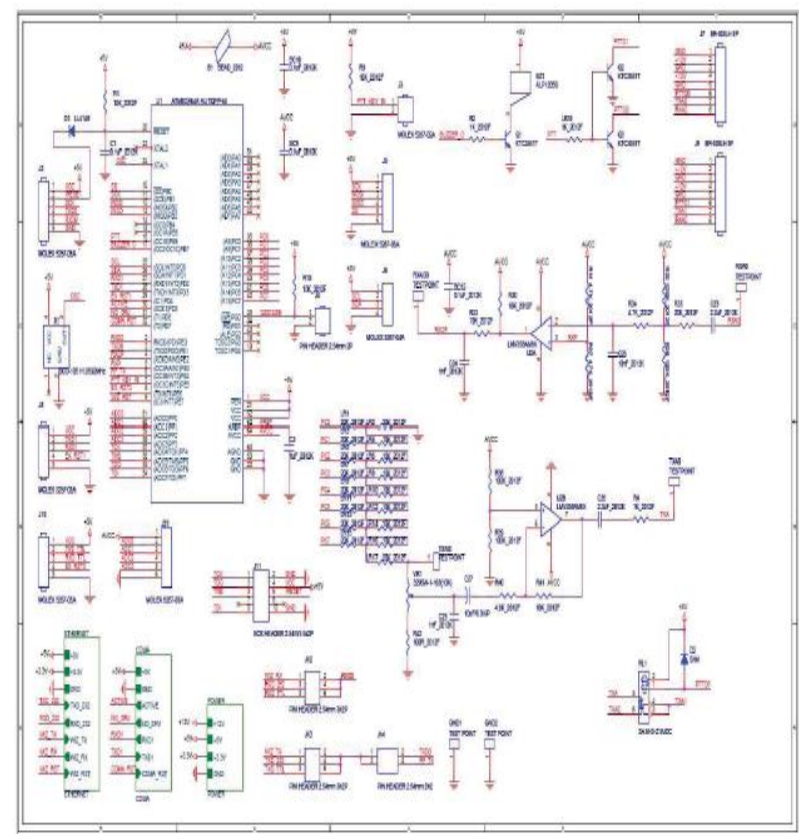

Fig. 7: Circuit Diagram of RTU System.

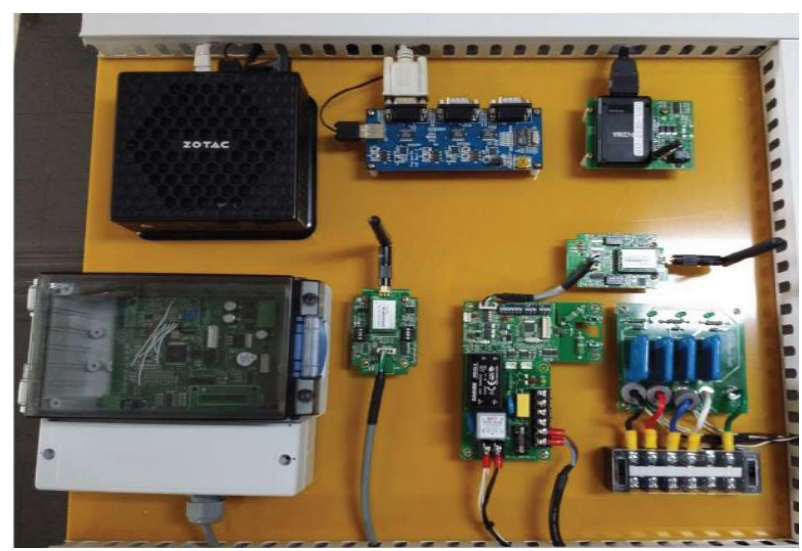

Fig. 8: Manufacture of Test Bed

\subsection{Building remote control system of street light pole based on web}

APM is composed of Apache, PHP, and MYSOL and it is easy to build web development environment in Window environment. Apach is a typical web server program and PHP in one of web programming langue that is easy to handle for beginner because variable data format is not too strict compared with JSP or ASP. Example of popular open source database is MYSQL. APM environment is built in tesr server. Screen of APM SETUP is shown in Figure 9. 


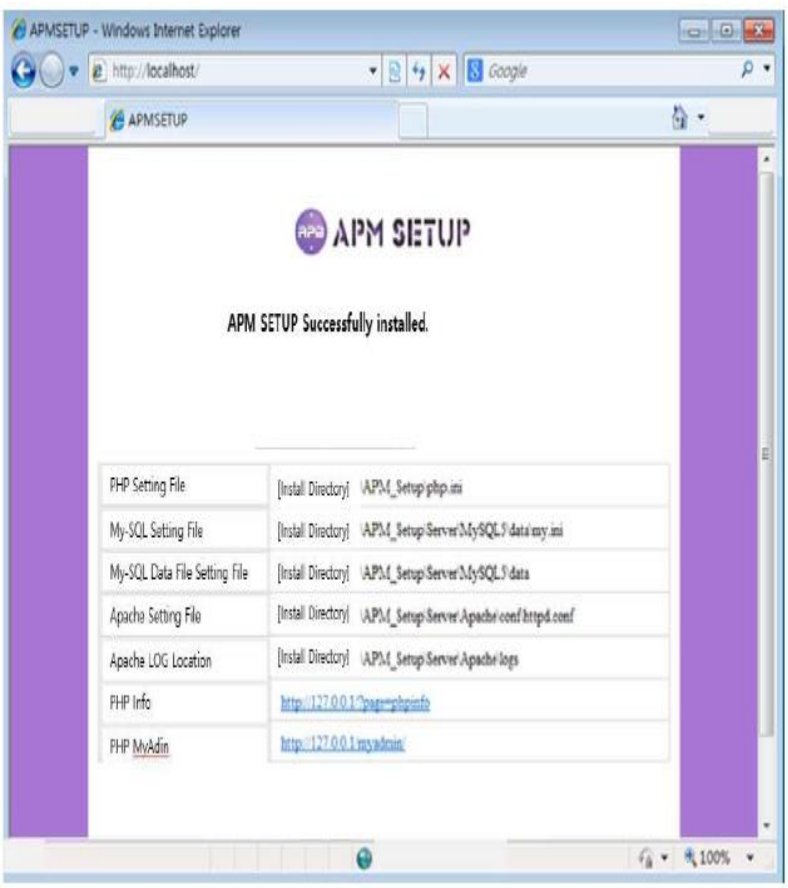

Fig. 9: Apm Setup Display.

\section{Conclusion}

The core technology of monitoring field in a solar power plant is secured through the breadth of technology development ranging from the consideration of remote control solution to the technology development, such as development of SPD counter for collecting information about thunderbolt in a solar power plant, development of RTU data module and its housing which is possible not only to transmit data but to collect it through Zigbee communication against SPD counter data, prior technology to implement smart integrated control system, and development of specialized technology for utilization of CDMA module.

\section{Acknowledgment}

This Study was conducted by research funds from Gwangju University in 2018 , Korea

\section{References}

[1] Ghobeity, A. , Noone, C. J. , Papanicolas, C. N. , Mitsos, A., Optimal time-invariant operation of a power and water cogeneration solarthermal plant, SOLAR ENERGY -PHOENIX ARIZONA THEN NEW YORK-.2011, 85(9), pp. 2295-2320.

[2] Andrade, G. A. , Pagano, D. J., Alvarez, J. D. , Berenguel, M., A practical NMPC with robustness of stability applied to distributed solar power plants, SOLAR ENERGY -PHOENIX ARIZONA THEN NEW YORK- . 2013, 92(-), pp.106-122.

[3] Woods, L.W. , Snow, S.W., The Impact of Telehealth Monitoring on Acute Care Hospitalization Rates and Emergency Department Visit Rates for Patients Using Home Health Skilled Nursing Care, Home healthcare nurse. 2013, 31(1), pp. 39-45.

[4] Wang, X., Gu, Y., Xiong, Z., Cui, Z., Zhang, T., Electronic Skin Silk-Molded Flexible, Ultrasensitive, and Highly Stable Electronic Skin for Monitoring Human Physiological Signals (Adv. Mater. 9/2014), Advanced materials. 2014, 26(9), pp. 1309-1309.

[5] Sugimoto, H., Lightning Protection against Winter Lightning, INTERNATIONAL CONFERENCE ON LIGHTNING PROTECTION. 2008, 28(1), pp. 26-32.

[6] Lynn, Barry H., The Usefulness and Economic Value of Total Lightning Forecasts Made with a Dynamic Lightning Scheme Coupled with Lightning Data Assimilation, Weather and forecasting. 2017, 32(2), pp. 645-663.
[7] Zheng, Y., Rinoshika, A., Fujimoto, S. , Three-dimensional wavelet analysis on turbulent structure of dune wake flow, INTERNATIONAL JOURNAL OF WAVELETS MULTIRESOLUTION AND INFORMATION PROCESSING. 2015, 13(6), pp. 15500451550045 .

[8] Cai, J.-J. , Liang, H.-Y. , Nonlinear Wavelet Density Estimation for Truncated and Dependent Observations, INTERNATIONAL JOURNAL OF WAVELETS MULTIRESOLUTION AND INFORMATION PROCESSING. 2011, 9(4), pp. 587-610.

[9] Morris, T., Vaughn, R. , Dandass, Y., A Retrofit Network Intrusion Detection System for MODBUS RTU and ASCII Industrial Control Systems, Proceedings of the Annual Hawaii International Conference on System Sciences. 2012, 45(3), pp. 2338-3425.

[10] Lee, J.-H. , Lee, S.-J. , An Accuracy Improvement on Acquisition Time of SCADA RTU Status Event, CHONGI HAKHOE NONMUNJI. 2013, 62(3), pp. 332-341. 